\title{
Non-Invasive Method of Diabetes Measurement using Teg Sensor via Foot Skin Temperature
}

\author{
R. Ganesan
}

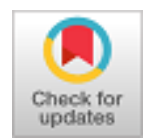

\begin{abstract}
Diabetes is a type of metabolic dieses identified by unstable blood glucose level due to the defect in body to generate or use of insulin. Diabetes is created due to the defect in metabolism of converting the glucose to energy in blood. Hyperglycaemia is a stage were glucose value in the body is greater than $140 \mathrm{mg} / \mathrm{dl}$ which leads to type 1 diabetes for the patient. Type 1 diabetes is caused due to lack of generation of insulin in human blood and type 2 diabetes is caused due to resistance to insulin action which leads to several other diseases like foot ulcer and sever wounds in human foot or other parts of the body. Early diagnosing of diabetes disease plays an important task in improving the standard of healthy living. Traditional methods of identifying diabetes does not provides effective results and the results are not more reliable. Temperature based diabetes diagnosing model is defined using TEG sensor to analyse the heat changes in human foot. Imbalanced glucose level affects the performance of nerves system which leads to slower response for temperature change in the foot surface. TEG sensor is used to measure the heat transfer in foot by applying cold water over foot. The rate of temperature changes in foot represents the level of diabetes caused in the patient body. The signals from TEG sensor was collected and processed using signal analysis algorithm using MATLAB software.
\end{abstract}

Keywords : TEG Sensor, Diabetes foot, nerves breakdown, heat changes, dyadic wavelet transform, Autocorrelation.

\section{INTRODUCTION}

$\mathrm{D}_{\mathrm{s}}$ iabetes is a metabolic disease, which is caused due to increase in blood glucose level. About $5.1 \%$ of global population was affected by diabetes disease. International Diabetes Federation shows that $12 \%$ of total expenditure was spent over the medication for diabetes, which is $5 \%$ of the total expenditure for health care. Diabetic care is one of the important issues faced by the developing countries. Due to the severe complication of the diabetes disease, the cost of medication increases. Increase in blood glucose level leads to nerve damage and damage in extremities of body, which leads to severe foot related problem. So early detection of diabetes plays important role to maintaining food cycle which helps to reduces the effect of diabetes disease. In current diagnosing system analyse the diabetic foot with

Revised Manuscript Received on December 30, 2019.

* Correspondence Author

Dr. R. Ganesan*, Department of Electronics Instrumentation Engineering, Saveetha Engineering College, Chennai, Tamil Nadu, India. e-mail:ganesanhod@gmail.com

(C) The Authors. Published by Blue Eyes Intelligence Engineering and Sciences Publication (BEIESP). This is an open access article under the CC BY-NC-ND license (http://creativecommons.org/licenses/by-nc-nd/4.0/) clinical sensory tools and the results of foot severity are mostly measured manually. To automate the process of diabetes diagnosis a new approach was followed to measure the heat of the human foot. Infrared thermograph and other techniques to measure the temperature of foot is under development to identify the destructions in foot plantar temperature disturbance.

The traditional method of identifying the diabetes foot was based on sensory testing on foot, which shows the sensation of the patient's foot. The result was diagnosed by the rate of sensory power on the affected foot of the patent. Some of the tests for analysing the foot sensation are Semmes-Weinstein monofilament testing pinprick sensation and vibration analysis. Most of the traditional testing methods lack of accuracy because of variability in the sensing pattern of the patients. So detection of foot diabetes is still lacking in diagnosis method. Thermal analysis over the patient foot is the quantitative method to analyse and diagnose the foot diabetes in early stages. By analysing the thermal response by the affected foot the level of damage due to diabetes can be measured. The diabetes disease reduces the thermal response of the foot thus by applying cold stress; recovery of heat by the foot gets longer when the patient is affected by diabetes disease. The recovery speed of the foot can be handled as a measure to scale the effect of diabetes disease in the patient body.

Based on the survey by World Health Organisation the diabetes patients in the developing countries was doubled every year. Diagnosing of early diabetes is impossible due to the lack of trained professionals in the developing countries. So the low cost, effective and false less diagnosing method is required to perform early diagnose of diabetes disease in the developing countries. The thermograph based diabetes diseases diagnosing method was introduced to perform low cost and effective diagnosis for early detection of diabetes in developing contries. Developing the low cost diagnosing system is a challenge task to analyse the thermal pattern of the patient foot.

The major research focuses over the foot of the patients, which are affected by the diabetic peripheral neuropathy. The patients affected by diabetes are most vulnerable to the neural damage. $50 \%$ of the patients affected by diabetes posses neural damage and $15 \%$ of them are affected by foot ulcer during their lifetime. Foot ulcer is the main reason for cause of lower extremity amputation to the patients affected with diabetes diseases. The way to find the advanced causes due to nerve damage or foot ulcer is to develop early detection system for diabetes disease.

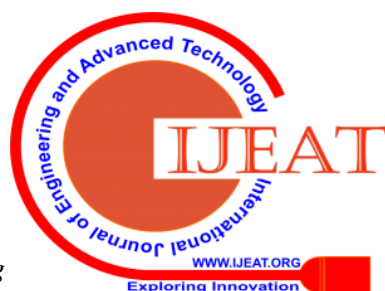


Traditional methods followed by the physicians may miss the detection of diabetes in the patient because of the low detection rate in the traditional methods. Only $60 \%$ of detection is possible through the traditional diagnosis methods. So the thermal analysis over the patient foot is developed to increase the accuracy of detection of diabetes disease in the early stage. The main theme of analysing the thermal effect of the foot by applying cold stress is followed and the heat recovery pattern is analysed by various thermographic systems. A peltier sensor based thermal analyser is proposed to analyse the thermal effect over the feet to analyse the cause of diabetes in the patient body.

\section{RELATED WORK}

Diabetes is a chronic disease which caused due to the lack of generation of insulin by pancreas or inefficient utilisation of insulin by human blood. This deficiency leads to type 1 diabetes and further leads to type 2 diabetes in human. Un treated condition of diabetes disease will result in severe diabetes retinopathy and increases the risk of early death. Thus, early detection of diabetes plays the vital role in health management to improve the quality of life. If the foot diabetes ia not early found it leads to foot amputation for diabetes patient. NIR images of foot was analysed to perform early diagnosis of diabetes. The thermo graphical behaviour of the patient foot will vary based on the level of diabetes in human blood. Pixel intensity matrix platform is used to analyse the NIR images acquired from the patient foot. [1]

A development kit with Infra Red sensor is developed to Thermograph and thermograph data's have been extracted from the patient's foot to perform diagnosis for foot diabetes. The method analyse the characteristics of heat over the foot surface by radiometric technique. Characteristic equation was framed to analyse each pixel of the sensor reading to analyse the heat flow in the patient foot. Three stages of analysis is performed they are thermal imaging, infra red senor reading and the bolometric sensor array. A matrix of heat flow is generated from the bolometric sensor. An artificial intelligence is applied to the system to analyse the foot. This method helps in detecting early diabetes using foot thermograph. [2] Data mining plays an important role in classification of medical images from large database system. Medical imaging system is one of the lead method used for health diagnosing by physicians. So image classification becomes more important in diagnosing the disease in health care application. Some of the image classification algorithms like k-mean clustering, neural network, Bayesian network and fuzzy logic are widely used in analysing the infected region on patient body. A system for analysing foot ulcer was developed to identify and classify the foot ulcer wound in patient's body. Diabetes is the major source for analysis and foot ulcer is the major threat in public health. Sevear diabetes in patients lead to foot ulcer, which generates long term, wound in the leg which leads to gangrene and may leads to death. Foot ulcer takes more time to heal it depends on the level of diabetes in patient body. Therefore, analysis of diabetes and foot ulcer helps the patients to over come the diabetes disease at early stage. [3] Measuring of acetone level in the human breath is the effective method of early diagnosis of diabetes and also used as a daily monitoring health complications like nerve dysfunctions, foot diabetes, analyse the effects of diabetes in the patient foot. sensor values to diagnose the effect of diabetes in the human

process for diabetes patients. Analysing along with other traditional methods like human blood analysing system the acetone analysis over human breath provides more merits like non invasive, inexpensive and more accurate. A nano sensor with K2W7O22 was developed to measure the acetone value from the human breath. The results shows that the nano sensor can effectively analyse the presence of acetone in human breath even at trace level at normal room temperature. Ferro electric and semiconducting technologies are adopted in nano sensor analysis to improve the detection rate of acetone in human breath. The level of acetone in human breath levels the presence of diabetes in the human blood. Nano sensor based diabetes analysis produces positive results which leads to development of smart devices to analyse the early stages of diabetes in the human body. [4]

Increase in the development of decision support system based on domain knowledge is an effective way to diagnose health issues like diabetes and heart diseases. Classical ontology does not provides more data for disease diagnosis but fuzzy ontology can generate effective data and knowledge about the diseases with uncertainty. Five layer fuzzy system was introduced to diagnose early diabetes in human. A fuzzy diabetes ontology is framed to generate knowledge about the diabetes with uncertainties. The five layers named fuzzy knowledge layer, group layer, group domain layer, relation layer and domain layer are generated to develop expert fuzzy system. Semantic decision support agent was developed along with the fuzzy system to develop efficient knowledge about the disease. [5] Data mining and machine learning approach is widely used methods for diagnosing diabetes disease. The effects of Type 2 diabetes can be controlled if the disease is diagnosed in the early stage itself. Support vector machine is implemented in diagnosing the diabetes disease in early stage. Along with support vector machine, an additional explanation module is added which converts the black box module of SVM to prediction tool of diabetes disease. A comprehensive rue set is developed to increase the accuracy, sensitivity and specificity of detection of diabetes. [6]Self-organising map is developed using non-invasive method by collecting information from the diabetes patients about self-care, condition of health and economic issues. Data analysis is performed to generate SOM, which produces two risk groups of developing diabetes foot. The groups provide knowledge about the present health condition and possibilities of occurrence of diabetes foot and the information collected is helpful in providing medical attention towards prevention of type 2 diabetes disease. Linear discrimination analysis is used to validate the correctness of the data collected in the groups through statistical analysis over dominant variable. [7] Diabetic foot ulcer is a sever disease caused due to improper diagnosis of type 2 diabetes disease which can leads to foot amputation. Foot ulcer can occur in any part of the leg in any shape, colour, and patterns based on the effect of ulcer in foot pathology. Current diagnosis methods for foot ulcer takes more time and cost to diagnose the effect of ulcer in the foot. Fully convolution network is framed to analyse the collected foot image to provide knowledge about the cause and effect of foot ulcer on diabetes patients. 705 images of foot ulcer images were provided to the network for effective diagnosing in clinical approach.

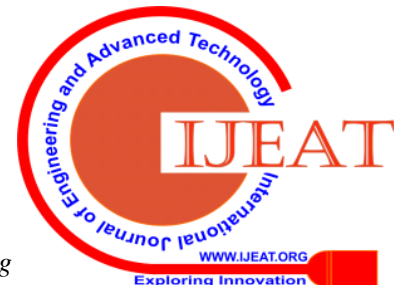


Two layer convolution network is developed to segment the ulcer cell from the surrounding normal skin from foot image. [8]. Support vector machine is applied to diagnose the type 2 diabetes disease. 50 to $80 \%$ of type 2 diabetes disease is unpredictable. The support vector machine along with the ensemble learning approach converts the black box decision making of SVM into transparent and comprehensive rules which also helps in handling imbalance problem. Ensemble learning algorithm provides rule sets with weight pecesion of $94.2 \%$ and hybrid system is provided to perform diagnosis over diabetes disease which provides the second opinion for lay users. [9]

A terahertz time domain spectroscopy is utilised to perform several degree deteriorisation over diabetes foot. This non-invasive method is used to monitor the hydration of skin of the affected user. [10]

A low cost medical embedded system is designed to monitor diabetic peripheral neuropathy. An infrared sensor is used to measure the reading of the heat flow in the foot in non contact way by applying cold stimulation to the foot of the patient. The rate of thermal recovery of the foot was measured to analyse the performance of foot response over the temperature change. This provides the rate of foot sensation over thermal change. This method helps to diagnose early occurrence of diabetic peripheral neuropathy and can be performed even in home, or any family care clinics were expensive test cannot be conducted. [11]

Sweat gland malfunctioning is an important symptom to diagnose pre diabetics in clinical manner. Sudoscan is a non-invasive device used to analyse the performance of the sudomotor by applying triangular electric pulses over the skin using nickel electrodes. The response of the skin for electric pulses is monitored and measures the electrochemical skin conductance. This provides more accurate on invasive method to analyse dysglycermia along with type 2 diabetes. The sensitivity of the Ni electrode is higher so that fasting of patients is not required. Vitro experiments and theoretical approach are performed to analyse the electrode behaviour and the electrochemical skin model. Electrochemical reactions and effects of several factors like property of ion channels in the seat duct is analysed using this method. [12]

Foot diabetes provides a economic burden over the quality of life providing physical impairments in patients life. Early detection of diabetes should be performed in order to overcome the occurrence of wound, ulceration and amputation. A cross sectional study over 44 volunteers with type 2 diabetes is performed. Foot plantar thermal images are collected from the participants using high-resolution infrared camera. Three segments of the foot are concentrated they are first finger, fifth finger and the heel. From the collected three results the output with higher temperature asymmetry is collected for analysing foot diabetes risk. The generated result is then compared with BMI and fat mass ratio of the concern patient. It shows that the BMI and body fat mass ratio plays the vital role in risk of type 2 diabetes in the patient. [13]

Type 2 diabetes increases the probability of cause of foot ulcer in patient foot. The foot ulcer may result in removing of total foot because the foot ulcer will totally damages the foot tissues may lead to early death. The foot ulcer can develop even applying improper pressure on the foot and also due to aging factor, gender and location of the patient residence. Chan vase algorithm is evolved to analyse the diabetes foot ulcer based on the foot temperature of the patient. The temperature of the patient foot is measured using IR thermal camera. [14]

Analysing of foot ulcer was performed through visual examination by the clinicians. And the analysis is mainly based on the size and healing status of the wound on the foot. Thus a quantitative and cost effective method is required to analyse the patients wound even by the caretakers in the home environment. This provides an active role in acceleration in healing of wound, reducing the travelling cost and reduces health care expenses. A wound image analysing system is developed to process the wound image taken from smart phone. Accelerated mean shift algorithm is utilised to perform image segmentation over images captured using smart phone. The outline of the foot is determined using the skin colour and the wound boundary was detected through connected region detection method. And the affected region within the wound boundary was analysed using red, yellow, black color evaluation model. The healing status of the wound is quantitatively assessed based on time records of the patient. [15]

\section{METHODOLOGY}

Early diagnosing of diabetes disease is an important research to reduce the health care cost due to several diseases caused due to diabetes in patients. The diabetes dieses initially leads to foot ulcer, wound and foot amputation, which may leads to loss of foot or leads to early death. A non-invasive and simple method is required for early diagnosis of diabetes even in patient friendly clinics or in home environment. Thermoelectric generator (TEG) sensor used to record the heat from the foot of the patients to diagnose level of diabetes in their body. The TEG sensor is placed on the left and right leg foot, rapid signals were collected, and the recorded signal is processed using discrete wavelet transformation and spectrum analysis algorithm. The result of algorithm was applied to regression model to calculate diabetes value from patients foot signal. Figure 1 show the block diagram of the method followed to measure diabetes value form patient foot using TEG Sensor.

The TEG sensor works using seebeck effect which converts the heat energy into electrical energy. The generation electrical energy varies based on the surface of the heat were it has been placed. The TEG sensor placed over the feet absorbs heat from the foot and the heat source is converted to electrical source. By measuring the generated electrical pulse heat on the foot can be measured. The TEG sensor is connected to instrumentation amplifier.

Instrumentation ampliferposses additional buffers than normal differential amplifiers. The TEG sensor and Instrumentation amplifier circuits are shown in figure $2 \& 3$. The additional buffers help to match the input impedance of the amplifier for every stage. Instrumentation amplifiers provides advantages like low off set voltage, higher common mode rejection ratio, high input resistance and high gain. 


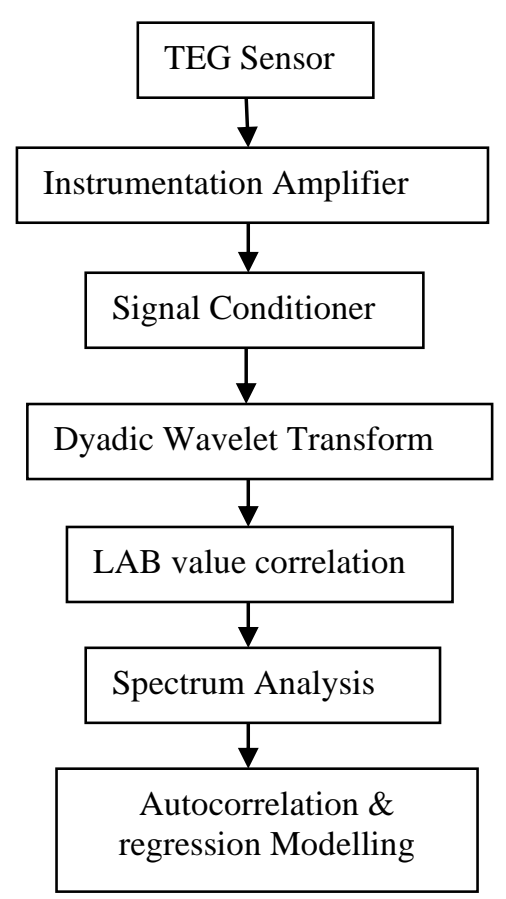

Figure 1: Block Diagram

Voltage gain (AV) $=\left(\frac{v_{0}}{v_{2}-v_{1}}\right)=\left(1+\frac{2 R_{1}}{R_{g}}\right) * \frac{R_{g}}{R_{2}}$

The output voltage generated from the TEG sensor will be low thus the voltage signal amplified using instrumentation amplifier and the output signal of the instrumentation amplifier is provided to signal conditioner circuit. The signal conditioner converts the signal into readable format of conventional instrumentation devices. The signal was then recorded using signal recording tools and applied to the MATLAB algorithms.

Dyadic Wavelet Transform

Dyadic wavelet transform divides the input into detailed coefficients and approximate coefficients. These splitted coefficients provide the knowledge about the high and low frequency signals and provides peak values of the low and high frequency signal from the input signal. Dyadic wavelet transform is a scaled sample of wavelet transform generated in the ratio of geometric sequence 2 . Time of the signal is not sampled. Reconstruction filter bank was used in dyadic wavelet transform.

The dyadic wavelet transform of signal $\mathrm{f}$ is defined as

$\mathrm{Wf}\left(\mathrm{u}, 2^{j}\right)=\int_{-\infty}^{+\infty} f(t) \frac{1}{\sqrt{2^{j}}} \varphi\left(\frac{t-u}{2^{j}}\right) d t=\mathrm{f} * \bar{\varphi}_{2^{j}}(u)$,

With

$$
\bar{\varphi}_{2^{j}}(t)=\varphi_{2^{j}}(-t)=\frac{1}{\sqrt{2^{j}}} \varphi\left(\frac{-t}{2^{j}}\right)
$$

If heisenberg covers all the input frequencies then it becomes the stable complete representation or else there exist A et B then

$$
\forall_{w} \in R, A \leq \sum_{j=-\infty}^{+\infty}\left|\hat{\varphi}\left(2^{j} w\right)\right|^{2} \leq B_{,}
$$

$L^{2} R_{\text {is }}$ the frame of dyadic wavelet family.

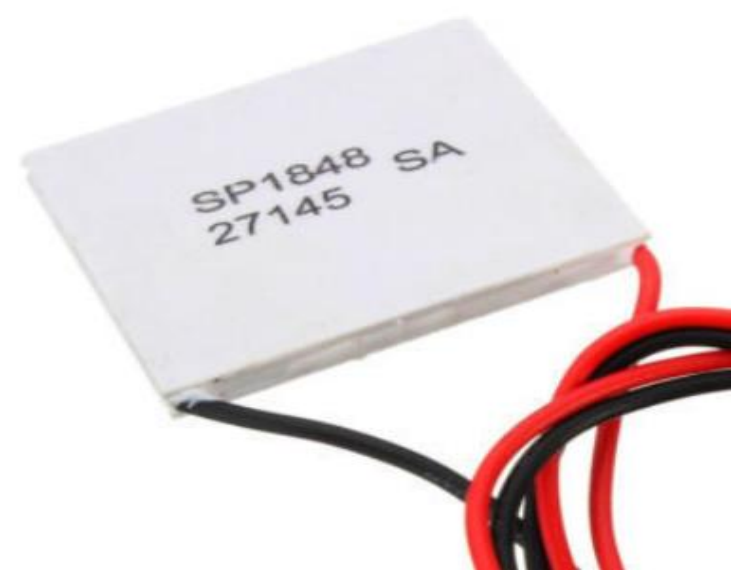

Figure 2 :TEG Sensor.

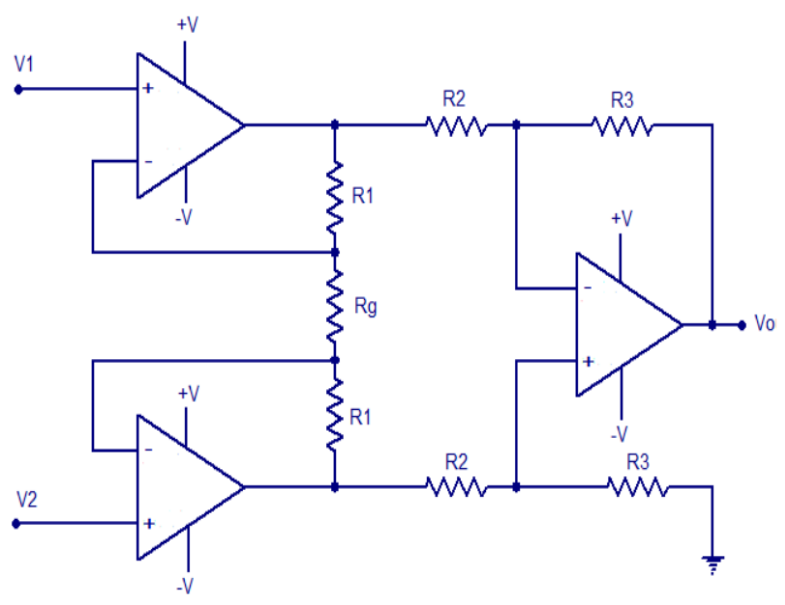

Figure 3: Instrumentation Amplifier

Orthogonal and biorthogonal wavelet basis along with perfect reconstruction filter banks are used to satisfy the previous conditions in the dyadic wavelet transform. Once the generated wavelet satisfies the previous conditions then fast dyadic wavelet transform along with reconstruction filter bank and scaling equation can be applied over input signal. Spectrum Analysis

Spectrum analysis is the process of determining the frequency contents from the continuous time signal with digital signal processing methods. It determines either energy spectrum or power spectrum of the input signal. $g_{a}(t)_{\text {is }}$ the input continuous time signal. $g_{a}(t)$ is band limited sufficiently, the discrete time equalent $g[n]$ should provide good relation with the input continuous time signal $g_{a}(t)$ Frequency spectrum analysis of the input signal provides valuable information based on the source from which the signal was recorded. Normally the signals are plotted with respect to time but in spectrum analysis the signal was plotted with respect to frequency. Analysing the amplitude, frequency and phase of the input signal is referred as frequency spectrum analysis of the signal. To extract these information from the input signal, the signal was analysed, digitized and filtered and plotted in frequency domain. The spectrum analysis provides the output results like spectrogram, magnitude response plot, probability distribution plot, autocorrelation plot.

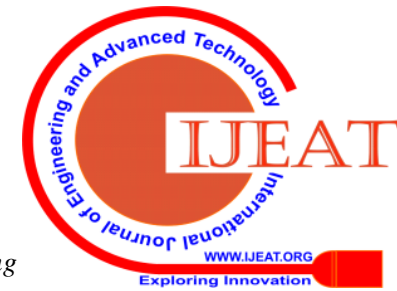


Spectrogram is the visual representation of the input signal in form of frequency, time and decibel rate. The frequency level in the time domain is represented in decibel. The concentration of information at particular frequency at particular time domain. The magnitude response plot represents the magnitude variations in frequency domain. When the magnitude of frequency $\mathrm{f}$ is in a complex form then the magnitude form is assigned as

$a(f)+i b(f)$.

then the magnitude response of frequency $f$ will be represented as

$\{[\mathrm{a}(\mathrm{f})] 2+[\mathrm{b} 9 \mathrm{f})] 2\} * 1 / 2$

Probability distribution plot is the statistical function which groups the values of the signal within the uniform surface area. This plot is utilised to find the range of the input information available in the raw value in space domain. Autocorrelation is used to compare the sequence signals of same input to analyse the repetition of same pattern within the input time sequence. It is used to overcome the loss due to noise present in the raw signal. These calculations are measured and plotted as graph which is discussed in result and discussion.

Regression Analysis

Regression analysis is the statistical model which is used to analyse the relationship between the variables from the segmented signal. The regression analysis is performed to analyse the relationship over the dependent variable and the independent variable. The peakvalues extracted form the frequencies of various sample signals are kept s dependent variables and the sigma value of the input signal was taken as independent variable. The level diabetes in the patient body was measured using regression analysis. The complet results collected from different patients sample was listed in table 1.

\section{RESULT \& DISCUSSIONS}

The TEG sensor was placed on right and left leg foot and series of signal are recorded. Before food and after food conditions are placed and the signals were recorded for six patients. The sigma, Mu, peak factor, dynamic range, autocorrelation and Diabetes range was recorded and shown in table 1.

The recorded unprocessed signal of right and left leg was shown in figure 4 and figure 5 . The heat flow pattern in right and left leg shows major difference in the amplitude. The recorded raw signal poses noise and unwanted signal along with the information signal. Thus, the raw signal was processed using dyadic transformation The dyadic wavelet transform was applied and the output of the transform is shown in figures 6 and 7. A dyadic decomposition and Haar wavelet transform was applied over the raw signal which pairs the input values, stores the difference and pass the sum values. This process was repeated continuously thus pairing up of sum increases to prove the next scale values which leads to $2^{n}-1$ differences and complete sum for the provided input signal. The unwanted amplitude shifts have been removed and the error less DWT signal was generated for processing.

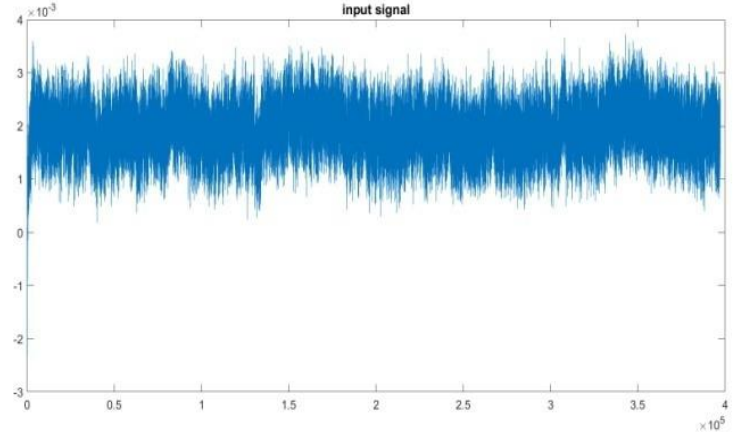

Figure 4 Patient 1 left foot

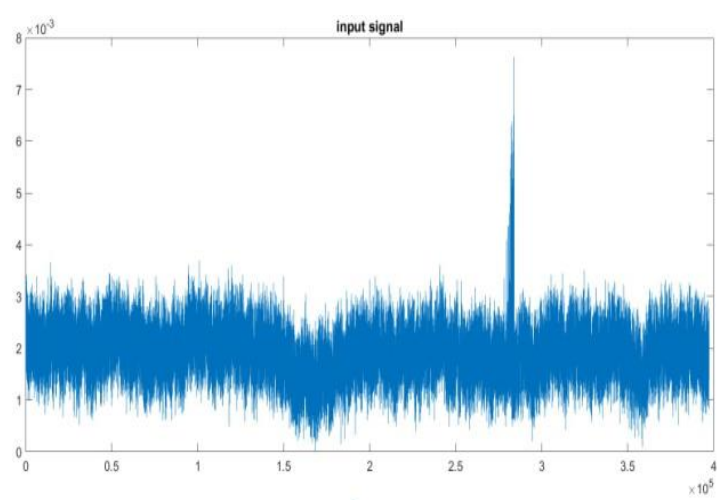

Figure 5 patient 1 right foot

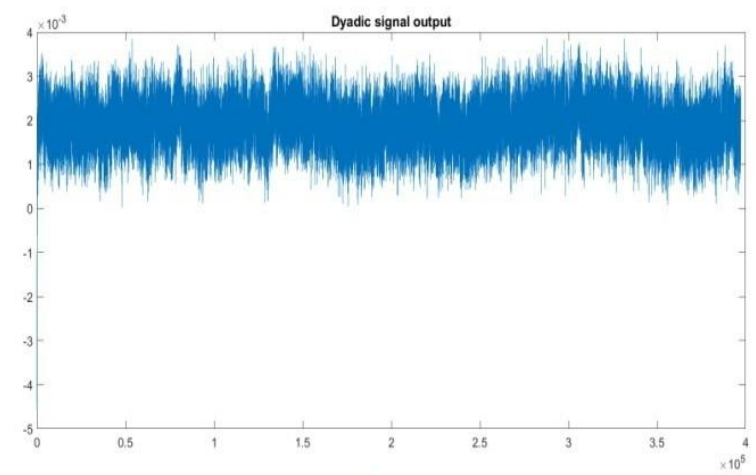

Figure 6 Dyadic transform over left foot

The output signal generated by the DWT is then analysed with spectrum analyser which generates spectrogram for the signal, autocorrelation, and probability plot. And digital values like $\mathrm{Mu}$, sigma, peak factor, dynamic range are generated which are listed in table1. The figure 8 and 9 shows the spectrogram outputs of the left and right leg DWT signals. The output is imaged in frequency time and decibel unit. The intensity frequency at time domain was plotted as an plot which shows the intensity of the signal at particular frequency range. Three-dimensional approach of signal visualisation provides more knowledge about the spreading of information over frequency domain. The probability plots are used to analyse weather the input values are normally distributed over a region. The figure 10 and 11 represents the normal probability plot of left and right leg. The probability plot of the left leg lies over the 0 to 0.4 layer and the probability plot of the right leg lies over 0.2 to 0.8 amplitude range. This difference in probability plot shows the difference in pattern of heat flow in the left and right leg of the patient.

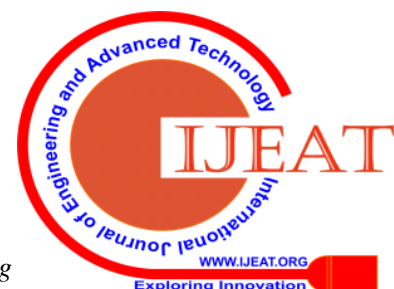


Non-Invasive Method of Diabetes Measurement using Teg Sensor via Foot Skin Temperature

\begin{tabular}{|c|c|c|c|c|c|c|c|c|}
\hline & & & Sigma & $\mathrm{Mu}$ & Peak factor(Q) & $\begin{array}{l}\text { Dynamic } \\
\text { Range(D) }\end{array}$ & Autocorrelation & Diabetes value \\
\hline \multirow[t]{4}{*}{ Patient 1} & $\begin{array}{l}\text { Left } \\
\text { foot }\end{array}$ & $\begin{array}{l}\text { Before } \\
\text { food }\end{array}$ & 0.1061 & 0.50683 & 5.7164 & 41.8684 & 176.7882 & 169.8338 \\
\hline & & $\begin{array}{l}\text { After } \\
\text { food }\end{array}$ & 0.1074 & 0.51644 & 5.5557 & 41.7272 & 176.6341 & 169.7576 \\
\hline & $\begin{array}{l}\text { Right } \\
\text { foot }\end{array}$ & $\begin{array}{l}\text { Before } \\
\text { food }\end{array}$ & 0.10159 & 0.53631 & 5.2585 & 24.2171 & 176.4995 & 170.098 \\
\hline & & $\begin{array}{l}\text { After } \\
\text { food }\end{array}$ & 0.054255 & 0.25069 & 11.8184 & 38.4164 & 177.1991 & 172.8715 \\
\hline \multirow[t]{4}{*}{ Patient 2} & $\begin{array}{l}\text { Left } \\
\text { foot }\end{array}$ & $\begin{array}{l}\text { Before } \\
\text { food }\end{array}$ & 0.053889 & 0.72966 & 2.714 & 6.2927 & 176.9709 & 172.893 \\
\hline & & $\begin{array}{l}\text { After } \\
\text { food }\end{array}$ & 0.093549 & 0.54824 & 5.0959 & 20.8934 & 176.5795 & 170.5692 \\
\hline & $\begin{array}{l}\text { Right } \\
\text { foot }\end{array}$ & $\begin{array}{l}\text { Before } \\
\text { food }\end{array}$ & 0.086425 & 0.56878 & 4.8019 & 15.2084 & 176.685 & 170.9866 \\
\hline & & $\begin{array}{l}\text { After } \\
\text { food }\end{array}$ & 0.08526 & 0.4416 & 5.9305 & 43.4637 & 175.6755 & 171.0549 \\
\hline \multirow[t]{4}{*}{ Patient 3} & $\begin{array}{l}\text { Left } \\
\text { foot }\end{array}$ & $\begin{array}{l}\text { Before } \\
\text { food }\end{array}$ & 0.10133 & 0.49495 & 5.9254 & 42.4115 & 176.5032 & 170.1133 \\
\hline & & $\begin{array}{l}\text { After } \\
\text { food }\end{array}$ & 0.10742 & 0.49396 & 11.5932 & 41.5109 & 176.8836 & 169.7564 \\
\hline & $\begin{array}{l}\text { Right } \\
\text { foot }\end{array}$ & $\begin{array}{l}\text { Before } \\
\text { food }\end{array}$ & 0.062084 & 0.25581 & 6.038 & 47.1967 & 176.6468 & 172.4128 \\
\hline & & $\begin{array}{l}\text { After } \\
\text { food }\end{array}$ & 0.11538 & 0.48548 & 2.9746 & 41.6557 & 177.0168 & 169.29 \\
\hline \multirow[t]{4}{*}{ Patient 4} & $\begin{array}{l}\text { Left } \\
\text { foot }\end{array}$ & $\begin{array}{l}\text { Before } \\
\text { food }\end{array}$ & 0.062243 & 0.70728 & 3.4038 & 7.4022 & 176.6627 & 172.4035 \\
\hline & & $\begin{array}{l}\text { After } \\
\text { food }\end{array}$ & 0.06982 & 0.67217 & 2.7959 & 13.7842 & 177.0514 & 171.9595 \\
\hline & $\begin{array}{l}\text { Right } \\
\text { foot }\end{array}$ & $\begin{array}{l}\text { Before } \\
\text { food }\end{array}$ & 0.056219 & 0.7226 & 3.3787 & 12.0821 & 176.9595 & 172.7565 \\
\hline & & $\begin{array}{l}\text { After } \\
\text { food }\end{array}$ & 0.07105 & 0.67401 & 5.1644 & 8.6799 & 177.0277 & 171.8875 \\
\hline \multirow[t]{4}{*}{ Patient 5} & $\begin{array}{l}\text { Left } \\
\text { foot }\end{array}$ & $\begin{array}{l}\text { Before } \\
\text { food }\end{array}$ & 0.097442 & 0.54313 & 5.8194 & 17.8025 & 176.3595 & 170.3411 \\
\hline & & $\begin{array}{l}\text { After } \\
\text { food }\end{array}$ & 0.10664 & 0.50049 & 5.579 & 27.6042 & 177.0382 & 169.8021 \\
\hline & $\begin{array}{l}\text { Right } \\
\text { foot }\end{array}$ & $\begin{array}{l}\text { Before } \\
\text { food }\end{array}$ & 0.10456 & 0.51558 & 5.3275 & 29.8272 & 177.4264 & 169.924 \\
\hline & & $\begin{array}{l}\text { After } \\
\text { food }\end{array}$ & 0.10165 & 0.53191 & 2.6558 & 23.5218 & 177.35 & 170.0945 \\
\hline \multirow[t]{4}{*}{ Patient 6} & $\begin{array}{l}\text { Left } \\
\text { foot }\end{array}$ & $\begin{array}{l}\text { Before } \\
\text { food }\end{array}$ & 0.060064 & 0.73411 & 6.2294 & 6.5419 & 176.9982 & 172.5312 \\
\hline & & $\begin{array}{l}\text { After } \\
\text { food }\end{array}$ & 0.10841 & 0.47593 & 2.7855 & 41.7272 & 175.9905 & 169.6984 \\
\hline & $\begin{array}{l}\text { Right } \\
\text { foot }\end{array}$ & $\begin{array}{l}\text { Before } \\
\text { food }\end{array}$ & 0.059544 & 0.7232 & 3.2551 & 6.8899 & 176.8764 & 172.5616 \\
\hline & & $\begin{array}{l}\text { After } \\
\text { food }\end{array}$ & 0.069189 & 0.68396 & 2.7895 & 13.3323 & 176.9427 & 171.9965 \\
\hline
\end{tabular}

Table 1: Results for 6 patients

The autocorrelation is applied to analyse the repeating patterns in the input signal. This process was mainly used to analyse the periodic signal that was hidden by the noise signal. In addition, used for finding the missing fundamental frequency from the signal hidden by the harmonic frequencies. The figure 12 and 13 sows the autocorrelation plots of left and right leg signal processed using dyadic wavelet transform. The autocorrelation plot was mapped with autocorrelation time in seconds and repeative sequence amplitude in unit 1.
The process was repeated for six more patents and the results were extracted using dyadic wavelet transform and signal analysing algorithm. The results were listed in table 1. The levels of diabetes in each patient was measured using regression mapping and listed in the table1. 


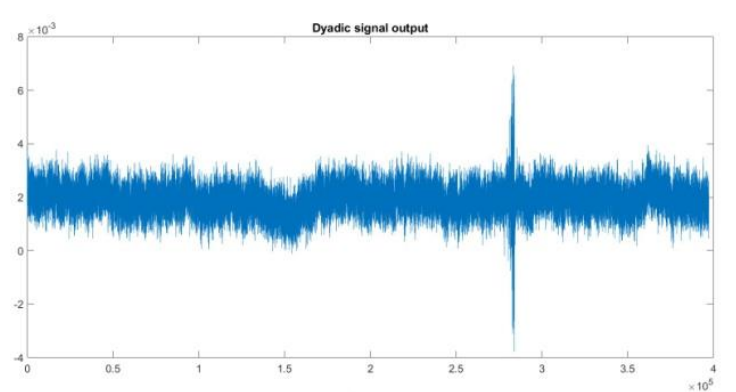

Figure 7 dyadic transform over right foot

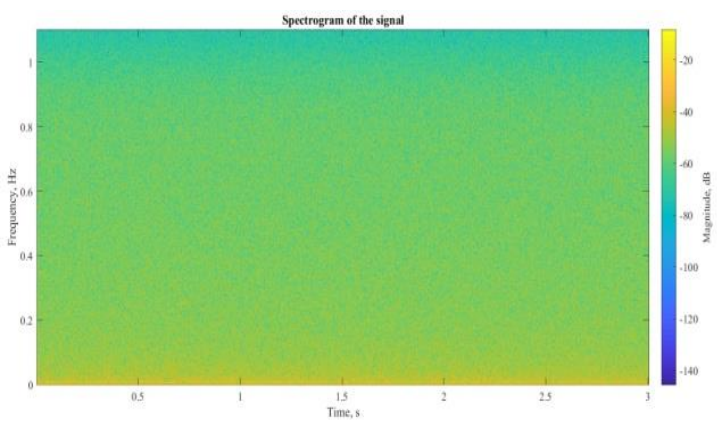

Figure 8 spectrogram of input signal of left leg

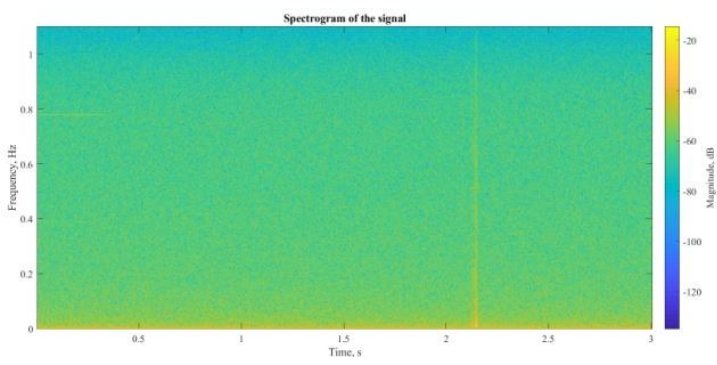

Figure 9 spectrogram of input signal of right leg

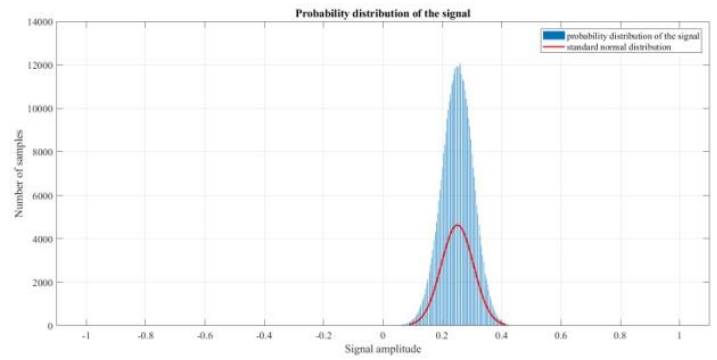

Figure 10 probability plot for left leg

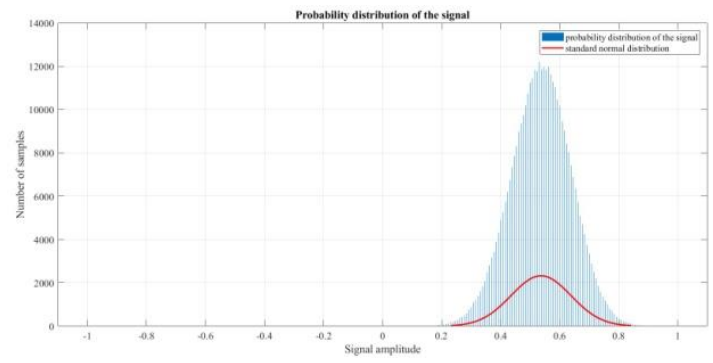

Figure 11 probability plot for right leg

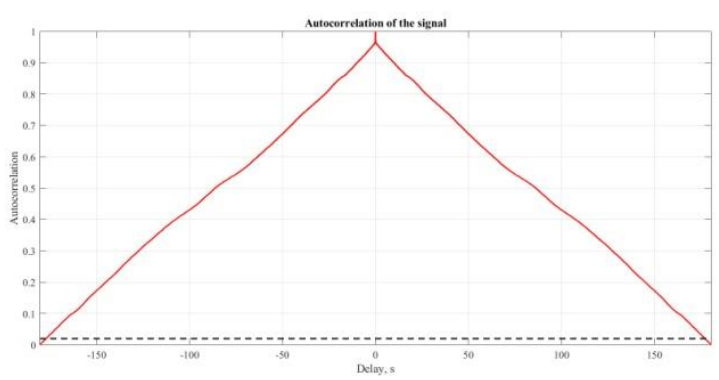

Figure 12 autocorrelation of the left leg input signal.

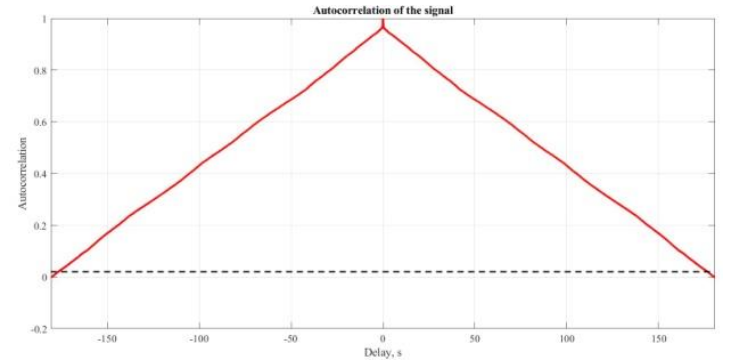

Figure 13 autocorrelation of the right leg input signal.

\section{CONCLUSION}

Diabetic is a major issues in public health. $50-80 \%$ of type 2 diabetes is undiagnosed by the traditional diagnosing methods. The aim of this work is to analyse the performance of early diagnosis of diabetic foot using TEG sensor. Major advantage of this system is non-invasive, simple to use and faster diagnosing. Wavelet and spectral analysis are performed over the recorded heat signal from the TEG sensor. The result generated using the algorithm provides clear recording of heat transfer in the human foot due to the effect of foot diabetes.

\section{REFERENCES}

1. P. M. Arabi, S. Nigudgi, T. Bhat, and A. Ahmed, "Investigations on diabetic foot impairment using NIR images and thermoregulatory behavior," 8th Int. Conf. Comput. Commun. Netw. Technol. ICCCNT 2017, pp. 4-8, 2017.

2. R. Bayareh, A. Vera, L. Leija, and J. Gutierrez-Martinez, "Programming of a system for the acquisition of images and thermographic data for the diabetic foot analysis," 2017 14th Int. Conf. Electr. Eng. Comput. Sci. Autom. Control. CCE 2017, pp. 2-8, 2017.

3. S. Patel, R. Patel, D. Desai, and D. Federation, "Diabetic Foot Ulcer Wound Tissue Detection and Classification," Int. Conf. Innov. Inf. Embed. Commun. Syst., pp. 1-5, 2017.

4. D. Wang, Q. Zhang, M. R. Hossain, and M. Johnson, "High Sensitive Breath Sensor Based on Nanostructured K2W7O22 for Detection of Type 1 Diabetes," IEEE Sens. J., vol. 1748, no. 11, pp. 4399-4404, 2018.

5. C.-S. Lee and M.-H. Wang, “A fuzzy expert system for diabetes decision support application.," IEEE Trans. Syst. Man. Cybern. B. Cybern., vol. 41, no. 1, pp. 139-153, 2011.

6. N. H. Barakat, A. P. Bradley, and M. N. H. Barakat, "Intelligible support vector machines for diagnosis of diabetes mellitus.," IEEE Trans. Inf. Technol. Biomed., vol. 14, no. 4, pp. 1114-1120, 2010.

7. A. C. B. H. Ferreira, B. M. Fernandes, and D. D. Ferreira, "Noninvasive Approach Based on Self Organizing Maps to Classify the Risk of Diabetic Foot," IEEE Lat. Am. Trans., vol. 16, no. 1, pp. 75-79, 2018.

8. M. Goyal, M. H. Yap, N. D. Reeves, S. Rajbhandari, and J. Spragg, "Fully convolutional networks for diabetic foot ulcer segmentation," 2017 IEEE Int. Conf. Syst. Man, Cybern., pp. 618-623, 2017. 
9. L. Han, S. Luo, J. Yu, L. Pan, and S. Chen, "Rule extraction from support vector machines using ensemble learning approach: An application for diagnosis of diabetes," IEEE J. Biomed. Heal. Informatics, vol. 19, no. 2, pp. 728-734, 2015.

10. G. G. Hernandez-Cardoso et al., "Development of a method of evaluation of diabetic foot deterioration by terahertz spectroscopic image," Int. Conf. Infrared, Millimeter, Terahertz Waves, IRMMW-THz, pp. 1-2, 2017.

11. G. Iven et al., "Non-contact Sensation Screening of Diabetic Foot Using Low Cost Infrared Sensors," 2014 IEEE 27th Int. Symp. Comput. Med. Syst., pp. 479-480, 2014.

12. K. Khalfallah et al., "Noninvasive galvanic skin sensor for early diagnosis of sudomotor dysfunction: Application to diabetes," IEEE Sens. J., vol. 12, no. 3, pp. 456-463, 2012.

13. E. B. Neves, A. J. Almeida, C. Rosa, J. Vilaca-Alves, V. M. Reis, and R. Mendes, "Anthropometric profile and diabetic foot risk: a cross-sectional study using thermography," 2015 37th Annu. Int. Conf. IEEE Eng. Med. Biol. Soc., pp. 1-3, 2015.

14. S. B. Vali, A. K. Sharma, and S. M. Ahmed, "Implementation of Modified Chan Vase Algorithm to Detect and Analyze Diabetic Foot Ulcers," 2017 Int. Conf. Recent Trends Electr. Electron. Comput. Technol., pp. 36-40, 2017.

15. L. Wang, P. C. Pedersen, D. M. Strong, B. Tulu, E. Agu, and R. Ignotz, "Smartphone-based wound assessment system for patients with diabetes," IEEE Trans. Biomed. Eng., vol. 62, no. 2, pp. 477-488, 2015.

\section{AUTHORS PROFILE}

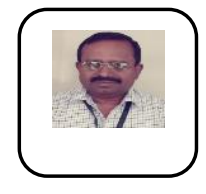

Dr.R.GANESAN, working as a Professor in Saveetha Engineering College, Chennai.I did B.E at Arulmigu Kalasalingam College of Engineering, M.E at MIT \& Ph.D at Anna University, Chennai, India. I possess 24 years of Teaching experience and 3 years of Industrial experience in Tuticorin Thermal Power Station. I am a Senior Member of ISTE, IETE \& MIE. I have chaired several Technical sessions \& acted as the moderator for many sessions in International Conferences. I have published 74 research papers in reputed International Journals and Registered a Patent. I am a recognished Supervisor in Anna University, Chennai. 